\title{
Hidden robust presence of a hole Fermi surface in a heavily electron-doped iron-based superconductor $\mathrm{LaFe}_{2} \mathrm{As}_{2}$
}

\author{
Hidetomo Usui $\oplus^{1}$ and Kazuhiko Kuroki ${ }^{2}$ \\ ${ }^{1}$ Department of Physics and Materials Science, Shimane University, 1060 Nishikawatsu-cho, Matsue, Shimane 690-8504, Japan \\ ${ }^{2}$ Department of Physics, Osaka University, 1-1 Machikaneyama-cho, Toyonaka, Osaka 560-0043, Japan
}

(Received 15 May 2019; published 15 October 2019)

\begin{abstract}
We investigate the electronic structure of a recently discovered, heavily electron-doped iron-based superconductor $\mathrm{LaFe}_{2} \mathrm{As}_{2}$. Although first-principles calculation shows apparent absence of hole Fermi surfaces around the $\Gamma$ point, we reveal, by hypothetically removing the La $d$ orbital contribution, that a hole Fermi surface around the $\Gamma$ point is essentially present. In the collapsed phase of $\mathrm{LaFe}_{2} \mathrm{As}_{2}$, which is nonsuperconducting, the hole Fermi surface is found to be absent, and the difference from the uncollapsed superconducting phase can be naturally understood within the spin-fluctuation-mediated pairing scenario.
\end{abstract}

DOI: 10.1103/PhysRevResearch.1.033025

\section{INTRODUCTION}

The Fermi surface (FS) configuration in the iron-based superconductors [1] has been an issue of great importance from the early stage of their study. This is because the FS consists of small electron and hole pockets (or cylinders in the three-dimensional sense), so it is sensitive to carrier doping, elemental substitution [2,3], and/or pressurization, and also because many theories suggest that the number and/or asymmetry of the electron and hole FSs play a key role in controlling the superconducting transition temperature $\left(T_{c}\right)$ and/or the pairing symmetry [4,5]. For instance, within the spin-fluctuation-mediated pairing scenario, Cooper pair scattering occurs between electron and hole FSs, so that too much carrier doping is expected to result in a disappearance of hole or electron FSs, and hence suppression or absence of superconductivity. $T_{c}$ dome obtained in a case when, e.g., electrons are introduced in $\mathrm{BaFe}_{2} \mathrm{As}_{2}$ by partially substituting Fe with Co $[6,7]$ is considered to be a manifestation of such a FS variance [8].

From this viewpoint, cases with heavy electron doping have attracted much attention. In $\mathrm{K}_{x} \mathrm{Fe}_{2-y} \mathrm{Se}_{2}$, a large amount of electrons are doped, and in fact, the angle-resolved photoemission spectroscopy (ARPES) experiments show that the hole bands sink below the Fermi level [9-11]. Still, $T_{c}$ is high, apparently implying that the hole bands are not playing an important role in the occurrence of superconductivity. A similar situation (with even a higher $T_{c}$ ) is observed in FeSe thin films grown on a substrate [12-14]. Partially motivated by these studies, the importance of the hole bands sinking below the Fermi level (the "incipient band") has been pointed out in

Published by the American Physical Society under the terms of the Creative Commons Attribution 4.0 International license. Further distribution of this work must maintain attribution to the author(s) and the published article's title, journal citation, and DOI. various studies [4,15-23]. On the other hand, another ARPES experiment on $\mathrm{K}_{x} \mathrm{Fe}_{2-y} \mathrm{Se}_{2}$ found that a hole band actually intersects the Fermi level [24]. Another series of material that is of great interest are the hydrogen-doped 1111 compounds such as $\mathrm{LaFeAsO}_{1-x} \mathrm{H}_{x}$ [25-30]. In these materials, high $T_{c}$ superconductivity survives up to large amounts of electron doping close to $50 \%$. Although such heavy electron doping is expected to wipe out the hole FS, some theoretical studies have pointed out that one of the hole bands (the $d_{x y}$ band) actually exhibits a non-rigid-band shift upon electron (hydrogen) doping, so that the hole band can in fact give rise to a FS and play an important role [25,31].

Given this controversial situation regarding heavily electron-doped iron-based superconductors, a recent discovery of a new superconductor $\mathrm{LaFe}_{2} \mathrm{As}_{2}$, exhibiting $T_{c}=$ $12.1 \mathrm{~K}$, opens up a renewed avenue in this hotly debated field [32]. In fact, considering $\mathrm{BaFe}_{2} \mathrm{As}_{2}$ as the mother (starting) compound, a complete substitution of $\mathrm{Ba}(+2)$ with $\mathrm{La}(+3)$ corresponds to a very heavy electron doping of $50 \%$. A band structure calculation of $\mathrm{LaFe}_{2} \mathrm{As}_{2}$ has shown that the hole FS around the $\Gamma$ point is indeed missing [32], which may be taken as natural considering the large amount of electron doping. This study also found that one electron FS interestingly possesses an unusual "jungle-gym-like" shape, whose role played in superconductivity, if any, is difficult to understand. It has also been found that the crystal structure transforms from a collapsed tetragonal structure to an uncollapsed tetragonal structure by annealing samples [32]. The superconductivity is found to emerge only in the uncollapsed phase, and no superconductivity has been observed in the collapsed phase. As discussed for other iron-based superconductors [4,33], it is expected that the difference in the electronic structure of collapsed and uncollapsed $\mathrm{LaFe}_{2} \mathrm{As}_{2}$ may hold the key to understanding the origin of superconductivity.

Here, we theoretically investigate the electronic structure of the uncollapsed and collapsed phases of $\mathrm{LaFe}_{2} \mathrm{As}_{2}$ to reveal its relevance to the origin of superconductivity. In the uncollapsed phase, we surprisingly find that the $d_{x y}$ cylindrical 


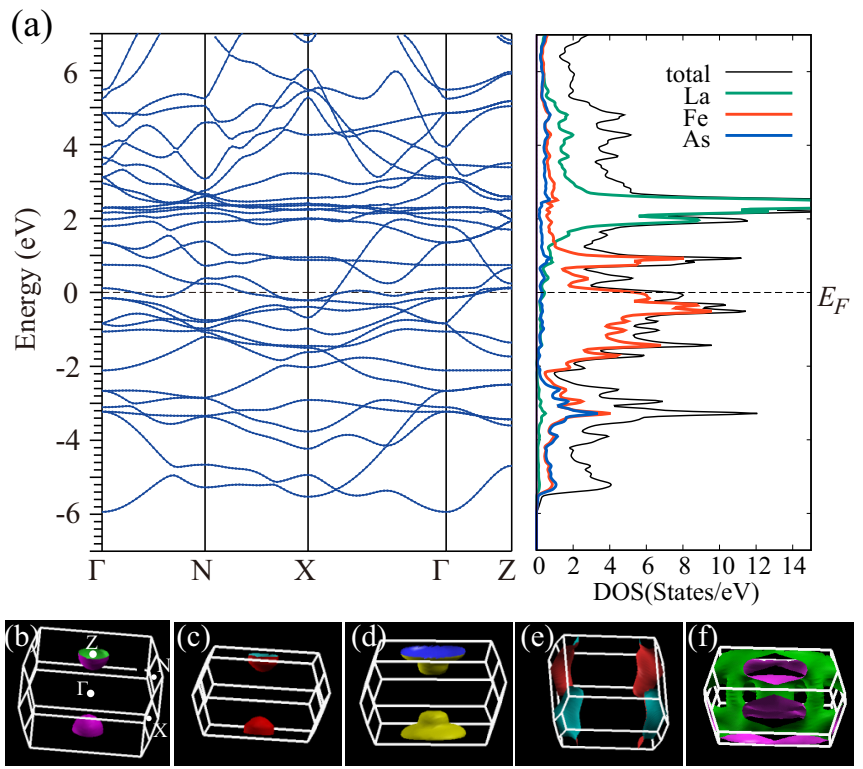

FIG. 1. (a) Electronic band structure and density of states of uncollapsed $\mathrm{LaFe}_{2} \mathrm{As}_{2}$. (b)-(f) Five FSs of $\mathrm{LaFe}_{2} \mathrm{As}_{2}$.

hole FS around the $\Gamma$ point, in the usual sense of the term used for the iron-based superconductors, can be considered as essentially present despite the heavy electron doping. Interestingly, both the survival of the $d_{x y}$ hole FS and its apparent absence can be traced back to the hybridization between the Fe $3 d$ and La $5 d$ orbitals. In the collapsed phase, on the other hand, the $d_{x y}$ hole FS is lost due to the large Fe-As-Fe bond angle. The correspondence between the presence or absence of the hole FS and the $T_{c}$ can be naturally understood within the spin-fluctuation-mediated pairing scenario.

\section{METHOD}

First-principles calculations are performed by means of the full-potential linearized augmented plane wave method as implemented in WIEN2K [34]. We use the Perdew-BurkeErnzerhof (PBE) exchange-correlation functional [35], and take $R K_{\max }=7$ and a $16 \times 16 \times 7 \mathrm{k}$ mesh for self-consistent calculation and a $22 \times 22 \times 22 k$ mesh for calculation of the density of states. We start with the electronic structure of the uncollapsed phase, whose lattice constants and the internal coordinates of the $\mathrm{Fe}$ atoms are given in Ref. [32].

\section{RESULTS AND DISCUSSION}

\section{A. Band structure of the collapsed phase}

The calculated band structure, the density of states, and the FS of the uncollapsed phase are shown in Fig. 1. In Fig. 1(a), it can be seen that the $\mathrm{Fe} d$ orbitals are dominant for the construction of the conduction bands around the Fermi level. As found in Ref. [32], the three hole and two electron FSs are obtained as shown in Figs. 1(b)-1(d) and 1(e)-1(f), respectively. The hole Fermi pockets and the cylindrical electron FS shown in Figs. 1(a)-1(e) are obtained, which can be seen in other iron-based superconductors. On the other hand, the

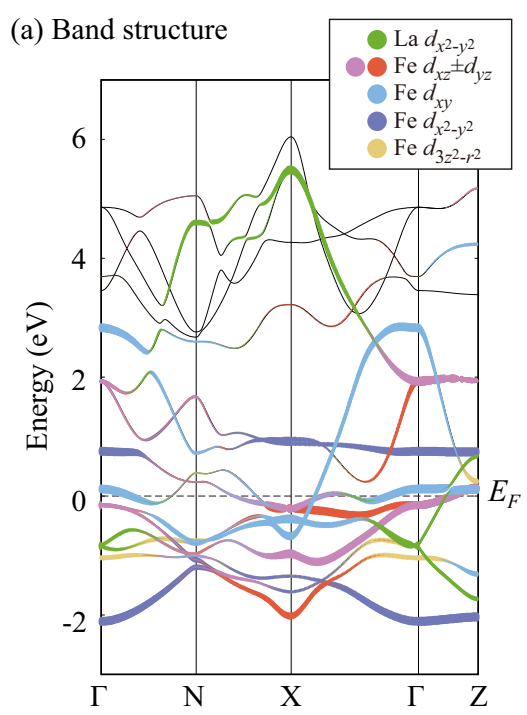

(c) Band structure of $\mathrm{BaFe}_{2} \mathrm{As}_{2}$

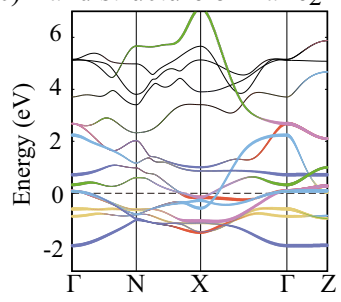

(b) Fermi surface

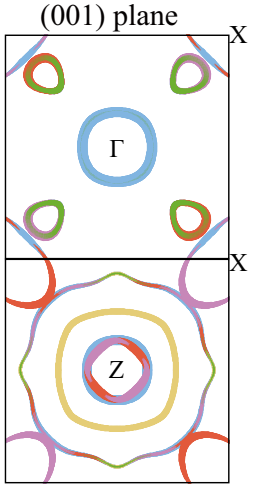

(110) plane

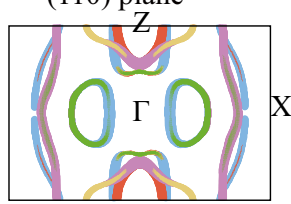

(d) Fermi surface of $\mathrm{BaFe}_{2} \mathrm{As}_{2}$

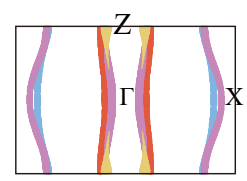

FIG. 2. (a) Band structure and (b) the FS of uncollapsed $\mathrm{LaFe}_{2} \mathrm{As}_{2}$ obtained from the 15-orbital tight binding model. (c) and (d) are the band structure and the FS of paramagnetic $\mathrm{BaFe}_{2} \mathrm{As}_{2}$, respectively, obtained in a similar way.

electron FS shown in Fig. 1(f) does not seem to correspond to any of the FSs seen in usual iron-based superconductors.

To understand the origin of this strange FS, we construct a 15-orbital tight binding model for $\mathrm{LaFe}_{2} \mathrm{As}_{2}$ exploiting the maximally localized Wannier functions of $d$ orbital projections centered at the $\mathrm{Fe}$ or La sites $(5 d$ orbitals $\times 3$ atoms $=15$ orbitals) using the WANNIER 90 package [36] and WIEN2WANNIER code [37]. The band structure and the FS of the tight binding model are shown in Figs. 2(a) and 2(b), respectively. In these figures, the orbital components of the $\mathrm{La} d_{x^{2}-y^{2}}$ and $\mathrm{Fe} d$ orbitals are expressed as colored circles. Other La $d$ orbitals have only small weight within the energy window presented here. For comparison, as shown in Figs. 2(c) and 2(d), we also perform similar calculation for $\mathrm{BaFe}_{2} \mathrm{As}_{2}$ (assuming a paramagnetic state) adopting the experimentally determined lattice structure [38].

First glance at the density of states of $\mathrm{LaFe}_{2} \mathrm{As}_{2}$ [Fig. 1(a)] might give an impression that the La orbitals do not strongly affect the band structure around the Fermi level. However, the La $d_{x^{2}-y^{2}}$ orbital weight is seen to be spread from -2 to $6 \mathrm{eV}$ measured from the Fermi level, namely, the La $d_{x^{2}-y^{2}}$ band crosses the Fermi level. In fact, if we look at the orbital weight in the band structure and the FS, we find that the La $d_{x^{2}-y^{2}}$ orbital does make a large contribution [see the green colored circles in Figs. 2(a) and 2(b)]. The jungle-gym-like shape of the FS therefore can be understood as originating from the 
(a) $\mathrm{LaFe}_{2} \mathrm{As}_{2}$ w/o La $d_{x^{2}-y^{2}}$

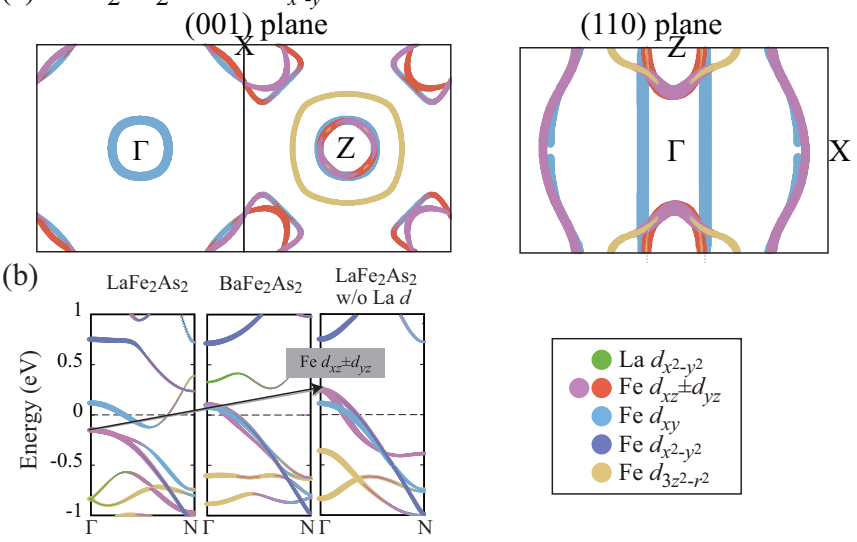

FIG. 3. (a) FS constructed from the 14-orbital tight binding model of uncollapsed $\mathrm{LaFe}_{2} \mathrm{As}_{2}$, where the $\mathrm{La} d_{x^{2}-y^{2}}$ orbital contribution is hypothetically removed. (b) Band structure of the 15-orbital models of uncollapsed $\mathrm{LaFe}_{2} \mathrm{As}_{2}$ (left) and $\mathrm{BaFe}_{2} \mathrm{As}_{2}$ (center), and the 10-orbital model of uncollapsed $\mathrm{LaFe}_{2} \mathrm{As}_{2}$ (right), where all the La $d$ orbitals are hypothetically removed from the 15-orbital model. The Fermi energy of the hypothetical models of $\mathrm{LaFe}_{2} \mathrm{As}_{2}$ is fixed at that of the original $\mathrm{LaFe}_{2} \mathrm{As}_{2}$.

hybridization between the Fe $3 d$ and La $d_{x^{2}-y^{2}}$ orbitals. This is why the strange shape is hardly seen in other iron-based superconductors. In fact, in $\mathrm{BaFe}_{2} \mathrm{As}_{2}$, nearly the entire $\mathrm{Ba}$ $d_{x^{2}-y^{2}}$ orbital weight lies above the Fermi level [Fig. 2(c)], so the $\mathrm{Ba} d_{x^{2}-y^{2}}$ orbital makes a small contribution on the FS. We note that the presence of a La $d$ orbital component around the Fermi level has also been discussed in $\mathrm{LaFe}_{2} \mathrm{P}_{2}$, which belongs to the same space group [39-41].

\section{B. Hidden hole Fermi surface}

Since the Fe $3 d$ orbitals are expected to play a main role in the occurrence of superconductivity, it is interesting to look at the "genuine Fe $3 d$ appearance" of the FS by hypothetically removing the La $d_{x^{2}-y^{2}}$ orbital contribution. For this purpose, we constructed a 14-orbital model, in which the hopping integrals among La $d_{x^{2}-y^{2}}$ and all the orbitals are neglected in the 15-orbital model. We adopt the same Fermi level as that of the 15-orbital model in order to directly compare the FSs with and without considering the La $d_{x^{2}-y^{2}}$ orbital contribution. The obtained FS is shown in Fig. 3(a). The hole FSs originating from the Fe $d_{x z / y z}$ orbitals are barely affected; they remain to be small pockets present only around the $Z$ point, consistent with the heavy electron doping. However, most interestingly, there appears a cylindrical hole FS around the $\Gamma-Z$ line, originating from the $\mathrm{Fe} d_{x y}$ orbital, whose volume is similar to that in $\mathrm{BaFe}_{2} \mathrm{As}_{2}$. We will later come back to the origin of this apparent contradiction with the heavy electron doping. If we turn to the FSs around the $X$ point, there appear two cylindrical electron sheets, similar to those seen in $\mathrm{BaFe}_{2} \mathrm{As}_{2}$. Coming back once again to the original FS in Fig. 2 from the above viewpoint, we can trace portions with strong Fe $d$ orbital weight to find that there essentially exist a $d_{x y}$ hole FS and a pair of electron FSs usually seen in 122 iron-based superconductors. The bottom line here is that there actually exist electron and hole cylindrical FSs originating from the $d_{x y}$ orbital, which can be favorable for spin-fluctuation-mediated superconductivity because this mechanism is based on the presence of electron and hole FSs originating from the same orbital.

The robust survival of the $d_{x y}$ hole FS against heavy electron doping by elemental substitution in the blocking layer apparently resembles the case of the hydrogen-doped 1111 systems [25,27,29,30] and is in contrast to the case of Co-doped $\mathrm{BaFe}_{2} \mathrm{As}_{2}$, where the elemental substitution takes place within the conducting layer, and only a rigid band shift of the Fermi level occurs as theoretically expected and experimentally observed [8,42]. For the hydrogen-doped 1111, it was theoretically shown in Ref. [31] that the substitution of oxygen with hydrogen leads to an increase of the positive charge in the blocking layer, which lowers the As $4 p$ level (moves away from Fe $3 d$ ) in the conducting layer. This in turn reduces the indirect electron hopping between nearest neighbor $\mathrm{Fe} 3 d_{x y}$ orbitals via As $4 p$, which raises the $d_{x y}$ hole band energy. One might expect a similar mechanism to work when $\mathrm{Ba}$ is substituted with $\mathrm{La}$ in $\mathrm{BaFe}_{2} \mathrm{As}_{2}$ because the positive charge in the blocking layer increases also in this case.

To see whether this is indeed the case, we construct 21orbital models for both $\mathrm{La}$ and $\mathrm{Ba}$ cases, where not only $\mathrm{Fe}$ $d$ and La $d$ but also As $p$ orbitals are explicitly considered. It is found that the on-site energies of both the As $p$ orbitals and the Fe $d$ orbitals are lowered by substituting $\mathrm{Ba}$ with La (for details, see the Appendix). In particular, the energy reduction of Fe $d$ orbitals and that of As $p_{z}$, through which the indirect $\mathrm{Fe}-\mathrm{Fe}$ hopping mainly occurs, is nearly the same about $0.1 \mathrm{eV}$, which implies that the robustness of the $d_{x y} \mathrm{FS}$ is not due to the same mechanism as in hydrogen-doped 1111 systems.

Since the answer to the puzzle must lie in the difference between $\mathrm{La}$ and $\mathrm{Ba}$, we go back to the 15-orbital model of $\mathrm{LaFe}_{2} \mathrm{As}_{2}$, and now hypothetically remove all the La $d$ orbitals to end up with a $15-5=10$ orbital model, whose band structure is shown in Fig. 3(b) (right). Interestingly, we find that the position of the Fe $d_{x y}$ and $d_{x z / y z}$ hole bands at the $\Gamma$ point is reversed by totally removing the La $d$ orbitals, namely, the energy level of the $d_{x z / y z}$ hole bands becomes higher. This means that the energy of the Fe $d_{x z / y z}$ hole bands are suppressed due to the mixing of the La $d$ orbitals. What is surprising here is that the role played by the weakly hybridized La $d$ orbitals other than $d_{x^{2}-y^{2}}$ (note that there is small weight of other La $d$ orbitals near the Fermi level) is important because removing just the La $d_{x^{2}-y^{2}}$ orbital does not give rise to $d_{x z / y z}$ hole FSs around the $\Gamma$ point as we have seen in the 14-orbital model [Fig. 3(a)]. If we compare $\mathrm{LaFe}_{2} \mathrm{As}_{2}$ and $\mathrm{BaFe}_{2} \mathrm{As}_{2}$ in Fig. 3(b), the energy level of the $d_{x z / y z}$ hole bands of the latter is higher than that of the former. This is because the on-site energy of the $\mathrm{Ba} d$ orbitals is higher than that of La $d$ (see Table I in the Appendix). Actually, similar analysis for $\mathrm{KFe}_{2} \mathrm{As}_{2}$ shows that the energy of the $d_{x z / y z}$ band becomes even higher with respect to $d_{x y}$ (not shown), because the energy level of the $\mathrm{K} d$ orbitals is higher than that of $\mathrm{Ba} d$. Our conclusion here is that the $d_{x y}$ FS remains upon heavy electron doping in $\mathrm{LaFe}_{2} \mathrm{As}_{2}$ because the doped electrons selectively enter the $d_{x z / y z}$ bands, whose energy is significantly lowered by the La $5 d$ hybridization [43]. 
(a) Band structure

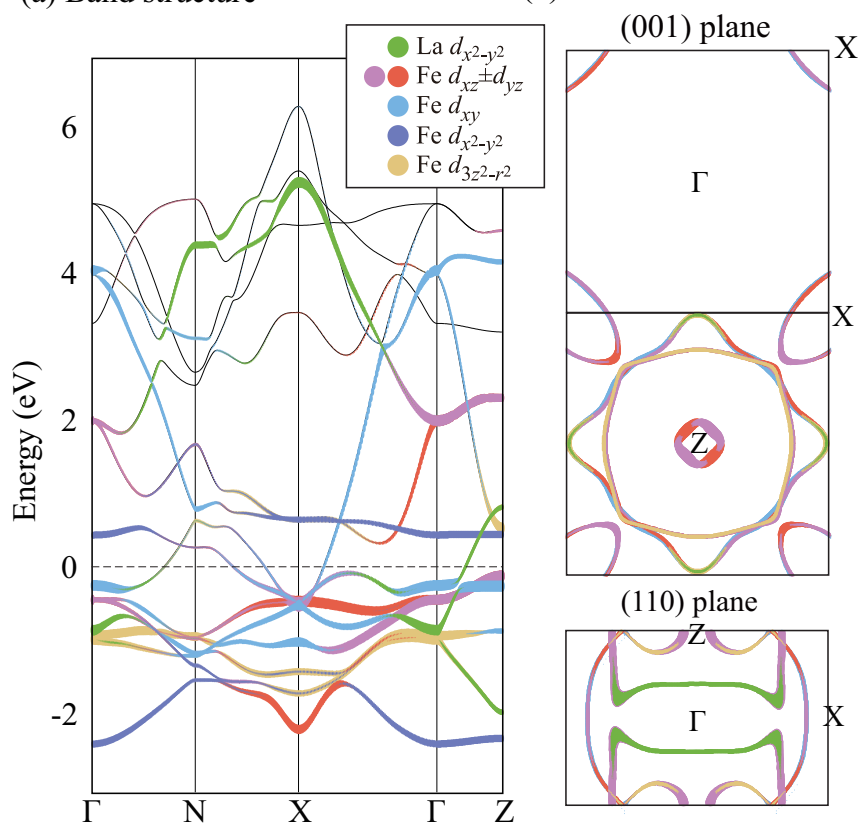

FIG. 4. (a) Band structure and (b) the FS obtained from a 15-orbital tight binding model of collapsed $\mathrm{LaFe}_{2} \mathrm{As}_{2}$.

\section{Comparison of the collapsed and uncollapsed phases}

Next, we will discuss the differences between the band structures of the uncollapsed and collapsed phases. Using the crystal structure of the collapsed phase described in Ref. [32], we calculate the band structure and FS as shown in Fig. 4. In the collapsed phase, the hole FS originating from the Fe $d$ orbitals almost disappears. In particular, the disappearance of the $d_{x y}$ hole FS, as compared to that in the uncollapsed phase, is due to the large Fe-As-Fe bond angle, namely, that of the uncollapsed phase is $110.8^{\circ}$, while that of the collapsed phase is $118.1^{\circ}$. For such a large bond angle, the $d_{x y}$ bands sink deeply below the Fermi level in the iron-based superconductors [44]. From the stand point of spin-fluctuation-mediated pairing, the absence of $d_{x y / y z / x z}$ hole bands is unfavorable for superconductivity, so the absence of superconductivity in the collapsed phase can be naturally understood from this viewpoint [45]. We also note that the band structure of collapsed $\mathrm{LaFe}_{2} \mathrm{As}_{2}$ is

TABLE I. Wannier spread $\Omega$ of the 21-orbital model of uncollapsed $\mathrm{LaFe}_{2} \mathrm{As}_{2}$.

\begin{tabular}{lcc}
\hline \hline & & \\
\hline Fe & $d_{z^{2}}$ & $\Omega\left(\AA^{2}\right)$ \\
& $d_{x z / y z}$ & 1.01 \\
& $d_{x y}$ & 1.20 \\
& $d_{x^{2}-y^{2}}$ & 1.26 \\
As & $p_{x / y}$ & 0.97 \\
& $p_{z}$ & 3.39 \\
La & $d_{z^{2}}$ & 3.45 \\
& $d_{x z / y z}$ & 4.43 \\
& $d_{x y}$ & 5.31 \\
& $d_{x^{2}-y^{2}}$ & 5.72 \\
& & 4.06 \\
\hline \hline
\end{tabular}

similar to that of $\mathrm{LaFe}_{2} \mathrm{P}_{2}$, where superconductivity also does not emerge $[39,40,46]$.

Finally, we comment on the superconducting gap structure of $\mathrm{LaFe}_{2} \mathrm{As}_{2}$ expected from the viewpoint of the orbital component on the FS. One may expect that the electronelectron interaction between the $\mathrm{Fe} 3 d$ and $\mathrm{La} 5 d$ orbitals are weak because they are spatially apart. Therefore, when the $\mathrm{Fe} 3 d$ orbitals play a main role in the occurrence of superconductivity, one can expect that the superconducting gap is small (or vanishing) around the portion of the FS where the La $d$ orbital character is strongly dominating. This may be one reason why $T_{c}$ is not as high as other 122 or 1111 compounds. Experimental probes are therefore expected to detect some kind of nodal feature in the gap structure.

\section{CONCLUSION}

In summary, we calculated the electronic structure of uncollapsed and collapsed $\mathrm{LaFe}_{2} \mathrm{As}_{2}$. In both cases, the unique $\mathrm{FS}$ is a consequence of the hybridization between the $\mathrm{Fe} 3 d$ and La $5 d$ orbitals. The main difference between the uncollapsed and collapsed phases is the presence of the $d_{x y}$ hole FS in the former, which is clearly revealed by hypothetically removing the La $d_{x^{2}-y^{2}}$ orbital contribution. The robustness of the $d_{x y}$ FS against heavy electron doping is due to the decrease of the on-site energy of the $\mathrm{La} / \mathrm{Ba} d$ orbitals when $\mathrm{Ba}$ is replaced with $\mathrm{La}$. On the other hand, the Fe-As-Fe bond angle is too large in the collapsed phase, resulting in the disappearance of the $d_{x y}$ hole FS. The correspondence between the absence or presence of the $d_{x y}$ hole FS and absence or presence of $T_{c}$ can be naturally understood within the spin-fluctuation-mediated pairing scenario. Assuming this scenario, there may be room for further increasing $T_{c}$ by optimizing the volume of the $d_{x y} \mathrm{FS}$ and/or removing the La $5 d$ orbital contribution, by, say, partially substituting the $\mathrm{La}$ and/or As atoms.

TABLE II. For uncollapsed $\mathrm{LaFe}_{2} \mathrm{As}_{2}$, the absolute values of the largest nearest neighbor hopping integrals $t$ from Fe to As, La to As, and $\mathrm{Fe}$ to $\mathrm{La}$ in each orbital. The nearest neighbor hoppings from $\mathrm{La}$ $d_{x^{2}-y^{2}}$ to Fe $d$ orbitals are also listed.

\begin{tabular}{|c|c|c|c|c|}
\hline \multicolumn{2}{|c|}{ From } & \multicolumn{2}{|c|}{ To } & \multirow{2}{*}{$\frac{|t|(\mathrm{eV})}{0.48}$} \\
\hline $\mathrm{Fe}$ & $d_{z^{2}}$ & As & $p_{z}$ & \\
\hline & $d_{x z / y z}$ & & $p_{x / y}$ & 0.60 \\
\hline & $d_{x y}$ & & $p_{z}$ & 0.63 \\
\hline & $d_{x^{2}-y^{2}}$ & & $p_{x / y}$ & 0.73 \\
\hline \multirow[t]{4}{*}{$\mathrm{La}$} & $d_{z^{2}}$ & As & $p_{z}$ & 0.48 \\
\hline & $d_{x z / y z}$ & & $p_{x / y}$ & 0.69 \\
\hline & $d_{x y}$ & & $p_{z}$ & 0.60 \\
\hline & $d_{x^{2}-y^{2}}$ & & $p_{x / y}$ & 0.40 \\
\hline \multirow[t]{7}{*}{$\mathrm{La}$} & $d_{z^{2}}$ & $\mathrm{Fe}$ & $d_{x z / y z}$ & 0.27 \\
\hline & $d_{x z / y z}$ & & $d_{z^{2}}$ & 0.29 \\
\hline & $d_{x y}$ & & $d_{x z / y z}$ & 0.05 \\
\hline & $d_{x^{2}-y^{2}}$ & & $d_{z^{2}}$ & 0.20 \\
\hline & & & $d_{x z / y z}$ & 0.06 \\
\hline & & & $d_{x y}$ & 0.08 \\
\hline & & & $d_{x^{2}-y^{2}}$ & 0.04 \\
\hline
\end{tabular}


TABLE III. Value of the on-site energy $(\mathrm{eV})$ measured from the Fermi energy for the 21-orbital models of uncollapsed $\mathrm{LaFe}_{2} \mathrm{As}_{2}$ and $\mathrm{BaFe}_{2} \mathrm{As}_{2} . \Delta_{\mathrm{La}-\mathrm{Ba}}$ is the energy difference between $\mathrm{LaFe}_{2} \mathrm{As}_{2}$ and $\mathrm{BaFe}_{2} \mathrm{As}_{2}$.

\begin{tabular}{lcccc}
\hline \hline & & $\mathrm{La}$ & $\mathrm{Ba}$ & $\Delta_{\mathrm{La}-\mathrm{Ba}}$ \\
\hline $\mathrm{Fe}$ & $d_{z^{2}}$ & -0.87 & -0.69 & -0.12 \\
& $d_{x z / y z}$ & -0.55 & -0.43 & -0.12 \\
& $d_{x y}$ & -0.61 & -0.49 & -0.12 \\
& $d_{x^{2}-y^{2}}$ & -0.87 & -0.78 & -0.09 \\
As & $p_{x / y}$ & -1.99 & -1.71 & -0.28 \\
& $p_{z}$ & -1.73 & -1.59 & -0.14 \\
$\mathrm{La} / \mathrm{Ba}$ & $d_{z^{2}}$ & 1.97 & 2.96 & -0.99 \\
& $d_{x z / y z}$ & 2.69 & 3.49 & -0.80 \\
& $d_{x y}$ & 2.59 & 3.33 & -0.73 \\
& $d_{x^{2}-y^{2}}$ & 1.83 & 2.74 & -0.92 \\
\hline \hline & & & &
\end{tabular}

\section{ACKNOWLEDGMENTS}

We acknowledge A. Iyo and $\mathrm{H}$. Mukuda for showing us the experimental results for $\mathrm{LaFe}_{2} \mathrm{As}_{2}$ prior to publication, and motivating us to start the present study. K.K. is supported by JSPS KAKENHI Grant No. JP18H01860.

\section{APPENDIX: ADDITIONAL INFORMATION ON THE La-Fe HYBRIDIZATION}

We present information regarding the mechanism of $\mathrm{La}-\mathrm{Fe}$ hybridization, which is the origin of the unique Fermi surface as revealed in the main text, and in particular, the role of the As $p$ orbitals played there. Namely, electrons can move from $\mathrm{Fe} 3 d$ to La $5 d$ orbitals through the direct hoppings from $\mathrm{Fe}$ to $\mathrm{La}$, but another possible path is via the indirect hoppings from Fe to La through As. In fact, the latter may play an important role because the nearest neighbor distance between $\mathrm{La}$ and $\mathrm{Fe}$ is larger than that between $\mathrm{Fe}$ and $\mathrm{As}$; the nearest neighbor distances are 2.39 $\AA$ for Fe-As, $3.20 \AA$ for La-As, and $3.53 \AA$ for Fe-La. In the main text, we have mainly discussed the band structure within the 15-orbital model constructed from the La $d$ and Fe $d$ Wannier orbitals, where the As $p$ orbitals are implicitly included in these Wannier orbitals. Since this model does not suffice to directly understand the role of As $p$ orbitals, here we have further investigated the role of the As $p$ orbitals by constructing a 21-orbital model, where we explicitly consider not only $\mathrm{La}$ and Fe $d$ but also As $p$ orbitals. Parameters obtained for the 21-orbital model are listed in Tables I-III. (Table III shows that the robustness of the $d_{x y}$ Fermi surface is not due to the same mechanism as in hydrogen-doped 1111 systems. See the main text for details.)

We first show the spread of the Wannier orbitals in the 21-orbital model in Table I. It is found that the average of the Wannier spreads (variances) is about $1.1 \AA^{2}$ for Fe $d$, $3.4 \AA^{2}$ for As $p$, and $5.0 \AA^{2}$ for La $d$ orbitals. This already

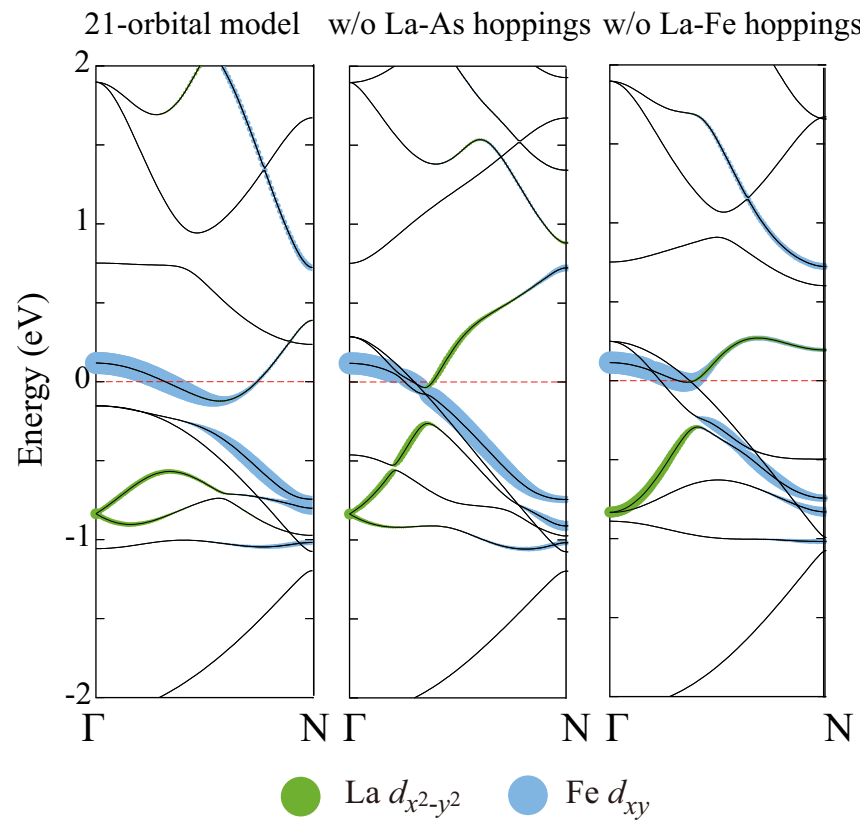

FIG. 5. Band structure and orbital component of the three 21orbital models of uncollapsed $\mathrm{LaFe}_{2} \mathrm{As}_{2}$; (left) the 21-orbital model constructed from the $\mathrm{Fe} d$, La $d$, and As $p$ orbitals, (center) the model without considering all the direct hoppings between La and As, and (right) the model without considering all the direct hoppings between $\mathrm{La}$ and $\mathrm{Fe}$.

suggests that the large Wannier spread of the La $d$ orbitals likely contributes to the hybridization between La $d$ and Fe $d$, considering the La-Fe distance of $3.53 \AA$. This is indeed seen in Table II, where the hoppings from La to Fe or La to As are found to be appreciable.

To clearly understand the effect of the indirect and direct hoppings on the band structure, we further construct two different 21-orbital models, where we neglect the hopping integrals between La and As, or between La and Fe. In Fig. 5, we compare the band structure of the three 21-orbital models. As discussed in the main text, the band splitting of the Fe $d_{x y}$ orbital around $-0.2 \mathrm{eV}$ originates from the La-Fe hybridization. When we switch off the hoppings between La and As, the band splitting still appears because of the direct hoppings. In addition, even if we do not consider La-Fe direct hoppings, the band splitting between the La and Fe $d$ orbitals exists because of the mixture through As $p$ orbitals. The magnitude of the band splitting without considering La-Fe and that without the La-As hoppings are almost the same, namely, the indirect and direct hoppings have almost the same contribution on the unique Fermi surface. Thus we conclude that the hybridization between La $d$ and $\mathrm{Fe} d$ orbitals originates from both the direct hoppings between $\mathrm{La}$ and $\mathrm{Fe} d$ orbitals and the indirect hoppings through the As $p$ orbitals.
[1] Y. Kamihara, T. Watanabe, M. Hirano, and H. Hosono, J. Am. Chem. Soc. 130, 3296 (2008).
[2] I. I. Mazin, D. J. Singh, M. D. Johannes, and M. H. Du, Phys. Rev. Lett. 101, 057003 (2008). 
[3] K. Kuroki, H. Usui, S. Onari, R. Arita, and H. Aoki, Phys. Rev. B 79, 224511 (2009).

[4] For a review, see, e.g., P. J. Hirschfeld, M. M. Korshunov, and I. I. Mazin, Rep. Prog. Phys. 74, 124508 (2011).

[5] For a review, see, e.g., H. Hosono and K. Kuroki, Physica C 514, 399 (2015).

[6] A. S. Sefat, R. Jin, M. A. McGuire, B. C. Sales, D. J. Singh, and D. Mandrus, Phys. Rev. Lett. 101, 117004 (2008).

[7] J.-H. Chu, J. G. Analytis, C. Kucharczyk, and I. R. Fisher, Phys. Rev. B 79, 014506 (2009).

[8] L. Fang, H. Luo, P. Cheng, Z. Wang, Y. Jia, G. Mu, B. Shen, I. I. Mazin, L. Shan, C. Ren, and H.-H. Wen, Phys. Rev. B 80, 140508(R) (2009).

[9] J. Guo, S. Jin, G. Wang, S. Wang, K. Zhu, T. Zhou, M. He, and X. Chen, Phys. Rev. B 82, 180520(R) (2010).

[10] T. Qian, X.-P. Wang, W.-C. Jin, P. Zhang, P. Richard, G. Xu, X. Dai, Z. Fang, J.-G. Guo, X.-L. Chen, and H. Ding, Phys. Rev. Lett. 106, 187001 (2011).

[11] X. H. Niu, R. Peng, H. C. Xu, Y. J. Yan, J. Jiang, D. F. Xu, T. L. Yu, Q. Song, Z. C. Huang, Y. X. Wang, B. P. Xie, X. F. Lu, N. Z. Wang, X. H. Chen, Z. Sun, and D. L. Feng, Phys. Rev. B 92, 060504(R) (2015).

[12] J. Shiogai, Y. Ito, T. Mitsuhashi, T. Nojima, and A. Tsukazaki, Nat. Phys. 12, 42 (2016).

[13] S. Tan, Y. Zhang, M. Xia, Z. Ye, F. Chen, X. Xie, R. Peng, D. Xu, Q. Fan, H. Xu, J. Jiang, T. Zhang, X. Lai, T. Xiang, J. Hu, B. Xie, and D. Feng, Nat. Mater. 12, 634 (2013).

[14] Q.-Y. Wang, Z. Li, W.-H. Zhang, Z.-C. Zhang, J.-S. Zhang, W. Li, H. Ding, Y.-B. Ou and P. Deng, K. Chang, J. Wen, C.-L. Song, K. He, J-F. Jia, S.-H. Ji, Y.-Y. Wang, L.-L. Wang, X. Chen, X.-C. Ma, and Q.-K. Xue, Chin. Phys. Lett. 29, 037402 (2012).

[15] H. Miao, T. Qian, X. Shi, P. Richard, T. K. Kim, M. Hoesch, L. Y. Xing, X.-C. Wang, C.-Q. Jin, J.-P. Hu, and H. Ding, Nat. Commun. 6, 6056 (2015).

[16] F. Wang, F. Yang, M. Gao, Z.-Y. Lu, T. Xiang, and D.-H. Lee, Europhys. Lett. 93, 57003 (2011).

[17] Y. Bang, New J. Phys. 16, 023029 (2014).

[18] X. Chen, S. Maiti, A. Linscheid, and P. J. Hirschfeld, Phys. Rev. B 92, 224514 (2015).

[19] Y. Bang, New J. Phys. 18, 113054 (2016).

[20] K. Kuroki, T. Higashida, and R. Arita, Phys. Rev. B 72, 212509 (2005).

[21] K. Matsumoto, D. Ogura, and K. Kuroki, Phys. Rev. B 97, 014516 (2018).

[22] K. Kobayashi, M. Okumura, S. Yamada, M. Machida, and H. Aoki, Phys. Rev. B 94, 214501 (2016).

[23] T. A. Maier, V. Mishra, G. Balduzzi, and D. J. Scalapino, Phys. Rev. B 99, 140504(R) (2019).

[24] M. Sunagawa, K. Terashima, T. Hamada, H. Fujiwara, T. Fukura, A. Takeda, M. Tanaka, H. Takeya, Y. Takano, M. Arita, K. Shimada, H. Namatame, M. Taniguchi, K. Suzuki, H. Usui, K. Kuroki, T. Wakita, Y. Muraoka, and T. Yokoya, J. Phys. Soc. Jpn. 85, 073704 (2016).

[25] S. Iimuraa, H. Okanishia, S. Matsuishib, H. Hirakac, T. Hondad, K. Ikedad, T. C. Hansene, T. Otomod, and H. Hosono, PNAS 114, E4354 (2017).

[26] K. Miyazawa, S. Ishida, K. Kihou, P. M. Shirage, M. Nakajima, C. H. Lee, H. Kito, Y. Tomioka, T. Ito, H. Eisaki, H. Yamashita,
H. Mukuda, K. Tokiwa, S. Uchida, and A. Iyo, Appl. Phys. Lett. 96, 072514 (2010)

[27] T. Hanna, Y. Muraba, S. Matsuishi, N. Igawa, K. Kodama, S. I. Shamoto, and H. Hosono, Phys. Rev. B 84, 024521 (2011).

[28] M. Hiraishi, S. Iimura, K. M. Kojima, J. Yamaura, H. Hiraka, K. Ikeda, P. Miao, Y. Ishikawa, S. Torii, M. Miyazaki, I. Yamauchi, A. Koda, K. Ishii, M. Yoshida, J. Mizuki, R. Kadono, R. Kumai, T. Kamiyama, T. Otomo, Y. Murakami, S. Matsuishi, and H. Hosono, Nat. Phys. 10, 300 (2014).

[29] S. Iimura, S. Matsuishi, H. Sato, T. Hanna, Y. Muraba, S. W. Kim, J. E. Kim, M. Takata, and H. Hosono, Nat. Commun. 3 943 (2012).

[30] S. Matsuishi, T. Maruyama, S. Iimura, and H. Hosono, Phys. Rev. B 89, 094510 (2014).

[31] K. Suzuki, H. Usui, S. Iimura, Y. Sato, S. Matsuishi, H. Hosono, and K. Kuroki, Phys. Rev. Lett. 113, 027002 (2014).

[32] A. Iyo, S. Ishida, H. Fujihisa, Y. Gotoh, I. Hase, Y. Yoshida, H. Eisaki, and K. Kawashima, J. Phys. Chem. Lett. 10, 1018 (2019).

[33] R. S. Dhaka, R. Jiang, S. Ran, S. L. Bud'ko, P. C. Canfield, B. N. Harmon, A. Kaminski, M. Tomić, R. Valentí, and Y. Lee, Phys. Rev. B 89, 020511(R) (2014).

[34] P. Blaha, K. Schwarz, G. K. H. Madsen, D. Kvasnicka, and J. Luitz, WIEN2K: An Augmented Plane Wave + Local Orbitals Program for Calculating Crystal Properties (Vienna University of Technology, Wien, 2001).

[35] J. P. Perdew, K. Burke, and M. Ernzerhof, Phys. Rev. Lett. 77, 3865 (1996).

[36] N. Marzari and D. Vanderbilt, Phys. Rev. B 56, 12847 (1997); I. Souza, N. Marzari, and D. Vanderbilt, ibid. 65, 035109 (2001); The Wannier functions are generated by the code developed by A. A. Mostofi, J. R. Yates, N. Marzari, I. Souza, and D. Vanderbilt (http://www.wannier.org/).

[37] J. Kunes, R. Arita, P. Wissgott, A. Toschi, H. Ikeda, and K. Held, Comput. Phys. Commun. 181, 1888 (2010).

[38] M. Rotter, M. Tegel, D. Johrendt, I. Schellenberg, W. Hermes, and R. Pöttgen, Phys. Rev. B 78, 020503(R) (2008).

[39] E. Mörsen, B. D. Mosel, W. Müller-Warmuth, M. Reehuis, and W. Jeitschko, J. Phys. Chem. Solids 49, 785 (1988).

[40] E. Razzoli, C. E. Matt, M. Kobayashi, X.-P. Wang, V. N Strocov, A. van Roekeghem, S. Biermann, N. C. Plumb, M. Radovic, T. Schmitt, C. Capan, Z. Fisk, P. Richard, H. Ding, P. Aebi, J. Mesot, and M. Shi, Phys. Rev. B 91, 214502 (2015).

[41] In Ref. [40], it was shown that $\mathrm{LaFe}_{2} \mathrm{P}_{2}$ and $\mathrm{CaFe}_{2} \mathrm{P}_{2}$ have nearly the same $\mathrm{Fe} 3 d$ occupation, namely, replacing $\mathrm{Ca}$ by $\mathrm{La}$ does not result in electron doping into the $\mathrm{Fe} d$ bands, but this does not apply to the relation between $\mathrm{LaFe}_{2} \mathrm{As}_{2}$ and $\mathrm{BaFe}_{2} \mathrm{As}_{2}$. Namely, although the hybridization with the $\mathrm{Fe} 3 d$ bands is stronger for the La $d$ bands than for the Ba $d$ bands, substituting $\mathrm{Ba}$ with La does result in electron doping, as can be seen from the comparison between Figs. 2(d) and 3(a), where the $d_{x z / y z}$ hole Fermi surfaces significantly shrink and the electron Fermi surfaces bulge in $\mathrm{LaFe}_{2} \mathrm{As}_{2}$.

[42] Z.-H. Liu, A. N. Yaresko, Y. Li, D. V. Evtushinsky, P.-C. Dai, and S. V. Borisenko, Appl. Phys. Lett. 112, 232602 (2018).

[43] The hybridization between La $d$ and Fe $d$ orbitals originates from both the direct hoppings between $\mathrm{La}$ and $\mathrm{Fe} d$ orbitals 
and the indirect hoppings through the As $p$ orbitals because of the large spread of the La $d$ orbitals (about $5 \AA$ ), despite the relatively large La-Fe nearest neighbor distance of $3.5 \AA$. For details, see the Appendix.

[44] H. Usui, K. Suzuki, and K. Kuroki, Supercond. Sci. Technol. 25, 084004 (2012).

[45] In our band calculation, we employed experimentally determined structural parameters. For the iron-based superconductors, it has been known that the experimentally observed structural parameters can be theoretically reproduced when the antiferromagnetic ordering is taken into account [e.g., I. I. Mazin, M. D. Johannes, L. Boeri, K. Koepernik, and D. J. Singh, Phys. Rev. B 78, 085104 (2008)]. Since our conclusion is that the Fermi surface configuration of $\mathrm{LaFe}_{2} \mathrm{As}_{2}$ is essentially similar to those of conventional iron-based superconductors, our band structure calculation can be considered as implicitly taking into account the magnetic effect in the employed structural parameters. In fact, recently, we came to know that I. I. Mazin, M. Shimizu, N. Takemori, and H. O. Jeschke in arXiv:1905.06190 show that the stripe-type antiferromagnetic order is required to reproduce the structural parameters of $\mathrm{LaFe}_{2} \mathrm{As}_{2}$ within DFT calculation.

[46] S. Blackburn, B. Prévost, M. Bartkowiak, O. Ignatchik, A. Polyakov, T. Förster, M. Côté, G. Seyfarth, C. Capan, Z. Fisk, R. G. Goodrich, I. Sheikin, H. Rosner, A. D. Bianchi, and J. Wosnitza, Phys. Rev. B 89, 220505(R) (2014). 